\title{
Untargeted serum metabonomics study of psoriasis vulgaris based on ultra-performance liquid chromatography coupled to mass spectrometry
}

\author{
Li Li ${ }^{1}$, Chuan-Jian Lu ${ }^{2}$, Ling Han ${ }^{1}$, Jing-Wen Deng ${ }^{2}$, Ze-Hui He ${ }^{3}$, Yu-Hong Yan ${ }^{2}$ and \\ Zhong-Zhao Zhang ${ }^{2}$ \\ ${ }^{1}$ Molecular Biology and Systems Biology Team of Chinese Medicine, Guangdong Provincial Hospital of Chinese Medicine, \\ Guangdong Provincial Academy of Chinese Medical Sciences, Guangzhou 510120, China \\ ${ }^{2}$ Department of Dermatology, Guangdong Provincial Hospital of Chinese Medicine, Guangdong Provincial Academy of Chinese \\ Medical Sciences, Guangzhou 510120, China \\ ${ }^{3}$ Large Data Research Team of Chinese Medicine, Guangdong Provincial Hospital of Chinese Medicine, Guangdong Provincial \\ Academy of Chinese Medical Sciences, Guangzhou 510120, China
}

Correspondence to: Li Li, email: Lily_vvv@163.com

Chuan-Jian LU, email: Luchuanjian888@vip.sina.com

Ling Han, email: Linghan36@163.com

Keywords: psoriasis; metabonomics; LC-MS; biomarker; high-throughput

Received: October 07, $2016 \quad$ Accepted: August 29, $2017 \quad$ Published: October 06, 2017

Copyright: Li et al. This is an open-access article distributed under the terms of the Creative Commons Attribution License 3.0 (CC BY 3.0), which permits unrestricted use, distribution, and reproduction in any medium, provided the original author and source are credited.

\section{ABSTRACT}

Psoriasis is a common, chronic, systemic inflammatory skin disease, the etiology and pathogenesis is unclear. An untargeted high-throughput metabonomics method based on liquid chromatography coupled to mass spectrometry was applied to study the serum metabolic changes in psoriasis vulgaris patients, and to discover serum potential biomarkers for identification, diagnosis and exploring pathogenesis of psoriasis. The serum metabolic profiles from 150 subjects (75 psoriasis patients and 75 healthy controls) were acquired, the raw spectrometric data were processed by multivariate statistical analysis, and 44 potential biomarkers were screened out and identified. The potential biomarkers were mainly involved in glycerophospholipid metabolism, sphingolipid metabolism, arachidonic acid metabolism, bile acid biosynthesis, indicated the pathogenesis of psoriasis may be related to the disturbed metabolic pathways.

\section{INTRODUCTION}

Psoriasis is a common, chronic, systemic inflammatory skin disease, the incidence and prevalence of psoriasis is significantly varied in different population, the prevalence in adults range from $0.91 \%$ (United States) to $8.5 \%$ (Norway), affecting approximately $2 \%$ of the population [1-3]. Patients with psoriasis are embarrassed about the appearance of their skin, involved in psychiatric comorbidity and suicide risk [4]. Psoriasis is considered a multisystem disease, the recent research results show it is associated with several comorbidities, such as inflammatory bowel disease, psychiatric disorders, metabolic syndrome and cardiovascular disease, as well as some new comorbidities in osteoporosis, obstructive sleep apnea and chronic obstructive pulmonary disease were reported in current literature [5]. Multiple epidemiologic studies and clinical researches have revealed that there was high prevalence of metabolic syndrome in patients with psoriasis compared with the general population or other skin diseases, and patients with more severe psoriasis have greater odds of metabolic syndrome than those with milder psoriasis. Psoriasis is associated with several metabolic diseases, including obesity, hypertension, diabetes mellitus, dyslipidemia, ischemic heart disease [6-11].

Psoriasis is a genetically determined by multiple genes, multi-environment factors stimulated, immune abnormalities induced, chronic inflammatory skin disease [12-14]. However, the etiology and pathogenesis of psoriasis is unclear, mainly associated with the genetic, 
immune, infection, metabolic disorders, endocrine, nervous and mental factor [15]. Metabolic abnormalities in patients with psoriasis had received extensive attention, involving proteins, lipids, carbohydrates, vitamins and enzymes and other metabolic disorders [16-23].

In recent years, research on etiology and pathogenesis of psoriasis mostly focus on immunological mechanisms and signaling pathways, the molecular mechanisms of psoriasis have been elucidated, the evidences of the relationship between psoriasis and metabolic syndrome come from clinical analysis or epidemiological analysis [24-26], however, only several studies dedicate to explore the global metabolic profiling of psoriasis patients [27-29].

Metabonomics is a modern high-throughput technique, it is an important component of systems biology, provides quantitative measures of global changes in the metabolic profiles of individuals in response to pathophysiological stimuli or genetic modification [30-32]. Profiling of metabolic products (metabolic phenotyping or metabotyping) has provided new insights into metabolic syndrome [33]. The metabonomics method has been applied to the diseases diagnosis [34], drug efficacy and toxicity evaluation [35-37], and disease pathogenesis exploration [38].

In this research, we used the ultra-performance liquid chromatography coupled with quadruple time of flight mass spectrometry (UPLC/Q-TOF MS) - based metabonomics strategy to investigate and compare the metabolic changes in the psoriasis vulgaris patients and healthy controls, as well as to discover the psoriasis biomarkers by identifying significantly changed metabolites.

\section{RESULTS}

\section{Metabolic profiling of the psoriasis vulgaris patient and healthy control were established by UPLC/Q-TOF MS}

The metabonomics study based on UPLC/Q-TOF MS was applied to investigate the serum metabolic profiling of the psoriasis patients and healthy controls. The typical serum base peak chromatograms in positive ion mode were shown in Figure 1.

\section{Methodology investigation}

The quality control (QC) sample obtained by mixing $20 \mu \mathrm{L}$ serum of each group of sample, processed as sample preparation. One QC sample was injected into LC-MS after every 10 serum samples running to ensure the repeatability and stability in the metabonomics raw data acquisition. The relative standard deviations (RSD) of the retention times of the main peaks was less than $1 \%$ and RSD of the main peak area corresponding value was less than $10 \%$ in the QC samples. The results showed that the metabonomics method had a good repeatability, the instrument stability was excellent, and the acquired data were reliable as well.
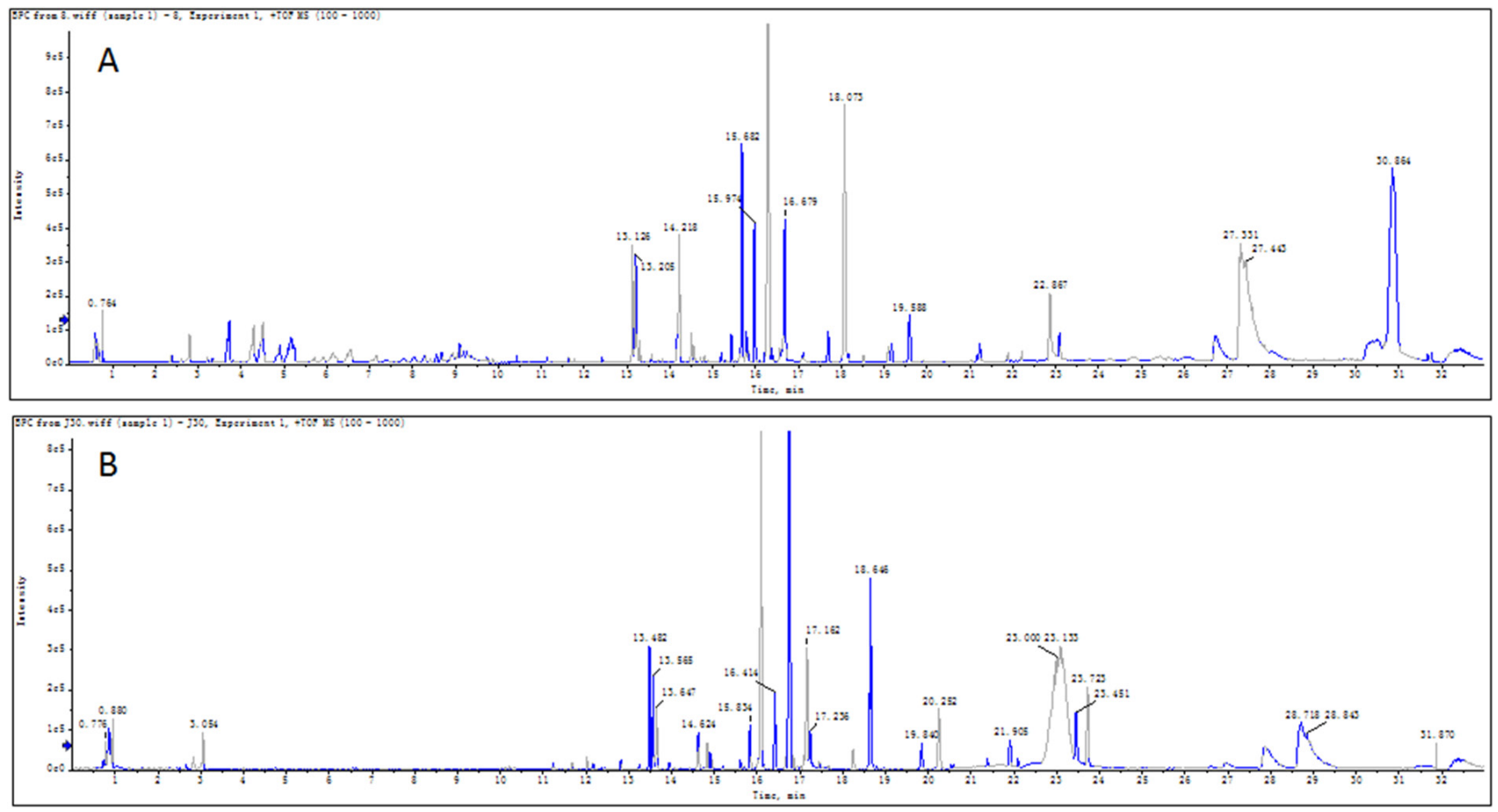

Figure 1: Typical base peak chromatograms (BPC) of serum sample. The typical base peak chromatograms based on UPLC/QTOF MS in positive ion mode of psoriasis patient (A) and healthy control (B) was shown respectively. 


\section{Multivariate statistical analysis}

After the raw data were acquired and processed, 5220 peaks were detected. The normalized data were imported to Simca-P for multivariate statistical analysis. Both unsupervised (PCA) and supervised (PLS-DA, OPLSDA) multivariate data analysis methods were applied to build the prediction models and classify the different groups. As an unbiased statistical approach, PCA was first carried out to distinguish the metabolic difference between PV and HC. Figure 2A showed the PCA score plots, there were distinct trends of separation between PV group and $\mathrm{HC}$ group indicated the inherent metabolic changes of PV individuals compared with the $\mathrm{HC}$ individuals. The PCA model showed the 17 principle components could explained $97.4 \%$ of the variation in the metabolic profiling $[R 2 X(\mathrm{cum})=0.974]$ with a predictability of $91.2 \%$ [Q2(cum) $=0.912]$, it was an excellent model. To further reveal the differences between PV and $\mathrm{HC}$ to discover significant variables, PLS-DA as a supervised method was applied. Figure 2B showed a clear separation in the
PLS-DA model between the two groups. The parameters demonstrated it was a satisfactory modeling, the $[\mathrm{R} 2 \mathrm{X}(\mathrm{cum})=0.774, \mathrm{R} 2 \mathrm{Y}(\mathrm{cum})=0.975, \mathrm{Q} 2$ (cum) $=0.971]$, this model predictive ability was excellent, and validated to be no over fitting by permutation test with 200 iterations (Figure 2D). The OPLS-DA model also validated to be no over fitting by permutation test (Figure 2E).

The severity of psoriasis is defined by PASI score, PASI $\leqq 10$ being considered mild, PASI $>10$ being considered moderate to severe, compared with the healthy control, statistical pattern recognition results were shown in Figure 3. Significant differences in metabolic status were observed among mild psoriasis group, moderate to severe psoriasis group, and healthy controls group, the three groups were clearly clustering, indicated that the severity of psoriasis was associated with the metabolism changes in serum. Thus it might be the key point of psoriasis occurrence and development process, we could discover the potential biomarkers to diagnose and cure psoriasis with more efficiency.
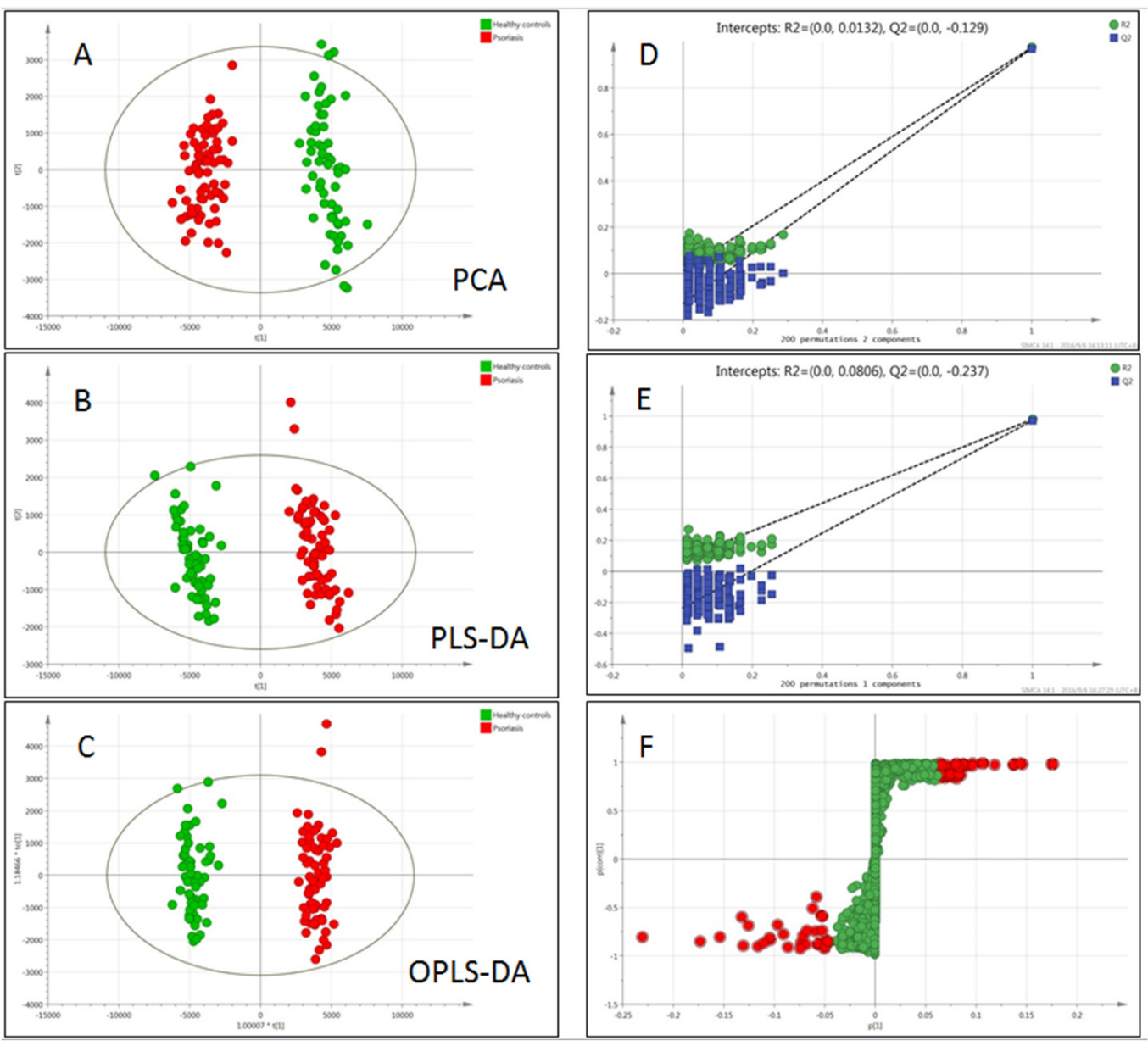

Figure 2: Multivariate data analysis and permutation test of psoriasis and healthy controls. (A) PCA scores plots, (B) PLSDA, (C) OPLS-DA(• PV patients, • HC), (D) Permutation test of PLS-DA, (E) Permutation test of OPLS-DA, (F) S-plots: The variables of the maximus VIP values were marked red. The normalized data were imported to Simca-P for multivariate statistical analysis. These models showed excellent predictive ability, and validated to be no over fitting by permutation test with 200 iterations. The red marked plots in the S-plot of PV and HC were significant different variables, and considered as the biomarker candidates. 


\section{Potential biomarker screening and identification}

According to the PLS-DA pattern recognition result, the Variable Importance in the Projection (VIP) values were sorted from small to large, the greater the value of grouping contribution is larger, combined with t-test, the significant difference $(\mathrm{P}<0.05)$ metabolites between the psoriasis patients group and healthy controls group can be screened out. In order to identify potential biomarkers among thousands of variables, an S-plot (Figure 2F) model was utilized, and the OPLS-DA model score plots $[\mathrm{R} 2$ (cum) $=0.814, \quad \mathrm{R} 2 \mathrm{Y}(\mathrm{cum})=0.979, \quad \mathrm{Q} 2$ (cum) $=0.972]$ were showed in Figure 2C. The S-plot of PV and HC was shown in Figure 2F, the marked plots were significant different variables, and considered as the biomarker candidates.

The TOF-MS can provide precise molecular weight of the screened metabolites, then search the potential biomarker in the human metabolome database (HMDB), combined with isotopic molecular weight, mass spectrometry ionization fragmentation patterns and biological significance, the potential biomarkers can be identified.
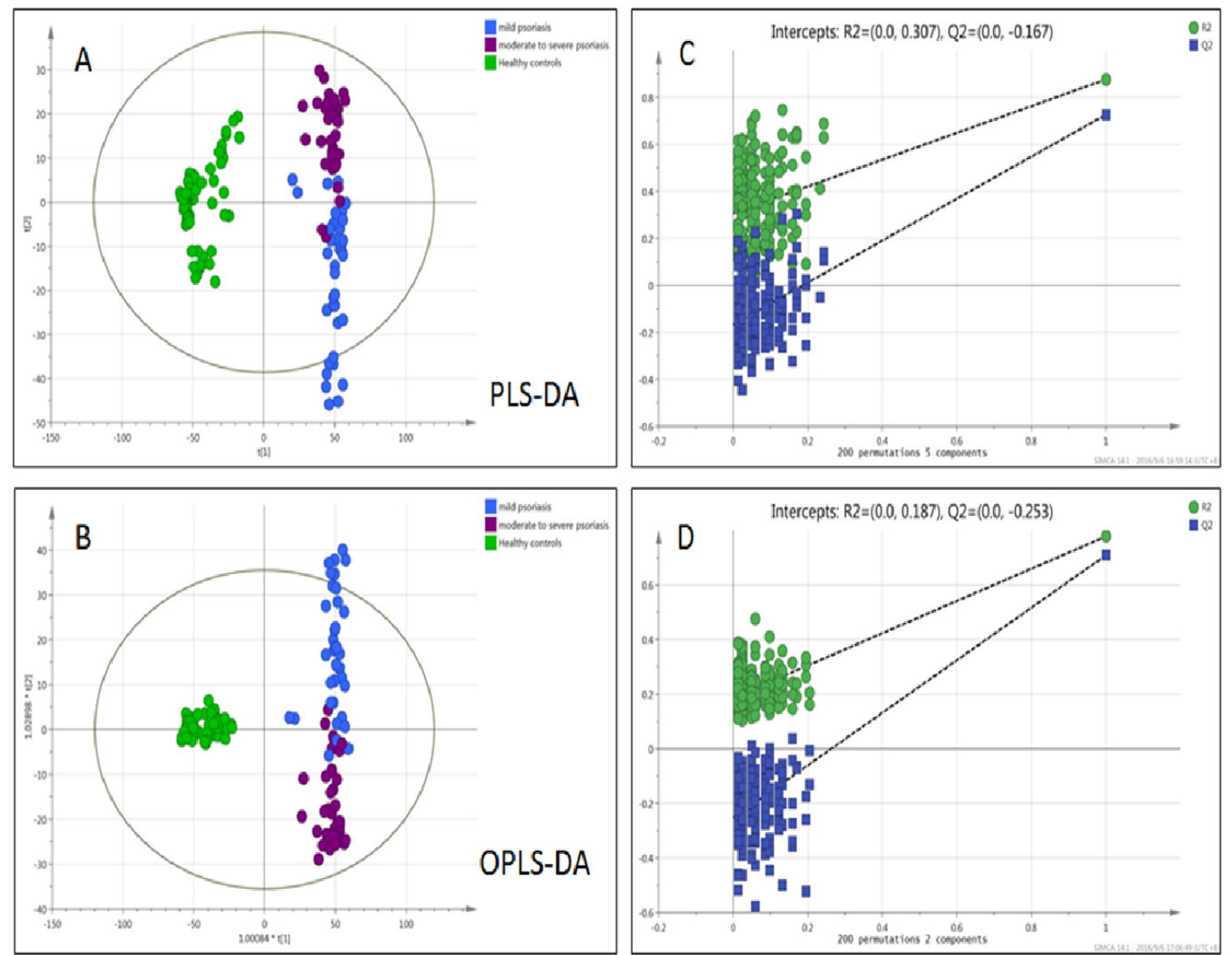

Figure 3: Multivariate data analysis and permutation test of psoriasis in varying degrees of severity. (A) PLS-DA scores plots, (B) OPLS-DA, (C) Permutation test of PLS-DA, (D) Permutation test of OPLS-DA ( $\bullet$ mild PV patients, $\bullet$ moderate to severe PV patients, $\bullet \mathrm{HC})$. The severity of psoriasis is defined by PASI score, PASI $\leqq 10$ being considered mild, PASI $>10$ being considered moderate to severe, compared with the healthy control, statistical pattern recognition results were shown. 
Table 1: Potential biomarkers and their metabolic pathways

\begin{tabular}{|c|c|c|c|c|c|c|c|c|c|c|}
\hline No. & $\mathbf{m} / \mathbf{z}$ & Identify Result & $\begin{array}{c}\text { Element } \\
\text { composing }\end{array}$ & Ion & HMDB ID & KEGG ID & Class & Fold Change & p-value & Trend $^{a}$ \\
\hline 1 & 497.293 & Leukotriene D4 & $\mathrm{C} 25 \mathrm{H} 40 \mathrm{~N} 2 \mathrm{O} 6 \mathrm{~S}$ & $\mathrm{M}+\mathrm{H}$ & HMDB03080 & $\mathrm{C} 05951$ & Fatty Acyls & 8.4 & $2.34 \mathrm{E}-46$ & $\uparrow$ \\
\hline 2 & 442.258 & Leukotriene E3 & $\mathrm{C} 23 \mathrm{H} 39 \mathrm{NO} 5 \mathrm{~S}$ & $\mathrm{M}+\mathrm{H}$ & HMDB02355 & - & Fatty Acyls & 6.9 & $5.55 \mathrm{E}-57$ & $\uparrow$ \\
\hline 3 & 627.442 & Eicosapentaenoic acid & $\mathrm{C} 20 \mathrm{H} 30 \mathrm{O} 2$ & $2 \mathrm{M}+\mathrm{Na}$ & HMDB01999 & $\mathrm{C} 06428$ & Fatty Acyls & 3.7 & $4.95 \mathrm{E}-43$ & $\uparrow$ \\
\hline 4 & 394.270 & $\begin{array}{l}\text { Dihomo-gamma- } \\
\text { Linolenoylethanolamide }\end{array}$ & $\mathrm{C} 22 \mathrm{H} 39 \mathrm{NO} 2$ & $\mathrm{M}+2 \mathrm{Na}-\mathrm{H}$ & HMDB13625 & $\mathrm{C} 13828$ & Fatty Acyls & 20.9 & $1.94 \mathrm{E}-80$ & $\uparrow$ \\
\hline 5 & 274.274 & Palmitic acid & $\mathrm{C} 16 \mathrm{H} 32 \mathrm{O} 2$ & $\mathrm{M}+\mathrm{NH} 4$ & HMDB00220 & C00249 & Fatty Acyls & 0.5 & 4.48E-39 & $\downarrow$ \\
\hline 6 & 396.273 & Prostaglandin E2 ethanolamide & $\mathrm{C} 22 \mathrm{H} 37 \mathrm{NO} 5$ & $\mathrm{M}+\mathrm{H}$ & HMDB13038 & - & Fatty Acyls & 21.9 & $6.82 \mathrm{E}-81$ & $\uparrow$ \\
\hline 7 & 806.565 & Lactosylceramide (d18:1/12:0) & $\mathrm{C} 42 \mathrm{H} 79 \mathrm{NO} 13$ & $\mathrm{M}+\mathrm{H}$ & HMDB04866 & C01290 & Sphingolipids & 0.5 & $9.96 \mathrm{E}-40$ & $\downarrow$ \\
\hline 8 & 302.305 & Sphinganine & $\mathrm{C} 18 \mathrm{H} 39 \mathrm{NO} 2$ & $\mathrm{M}+\mathrm{H}$ & HMDB00269 & $\mathrm{C} 00836$ & Sphingolipids & 0.4 & $6.84 \mathrm{E}-56$ & $\downarrow$ \\
\hline 9 & 542.322 & LysoPC (20:5) & $\mathrm{C} 28 \mathrm{H} 48 \mathrm{NO} 7 \mathrm{P}$ & $\mathrm{M}+\mathrm{H}$ & HMDB10397 & $\mathrm{C} 04230$ & Glycerophospholipids & 0.4 & $2.15 \mathrm{E}-47$ & $\downarrow$ \\
\hline 10 & 496.340 & LysoPC (16:0) & $\mathrm{C} 24 \mathrm{H} 50 \mathrm{NO} 7 \mathrm{P}$ & $\mathrm{M}+\mathrm{H}$ & HMDB10382 & $\mathrm{C} 04230$ & Glycerophospholipids & 0.6 & $1.28 \mathrm{E}-28$ & $\downarrow$ \\
\hline 11 & 524.373 & LysoPC (18:0) & $\mathrm{C} 26 \mathrm{H} 54 \mathrm{NO} 7 \mathrm{P}$ & $\mathrm{M}+\mathrm{H}$ & HMDB10384 & $\mathrm{C} 04230$ & Glycerophospholipids & 0.9 & $6.83 \mathrm{E}-06$ & $\downarrow$ \\
\hline 12 & 522.358 & LysoPC (18:1) & $\mathrm{C} 26 \mathrm{H} 52 \mathrm{NO} 7 \mathrm{P}$ & $\mathrm{M}+\mathrm{H}$ & HMDB02815 & $\mathrm{C} 04230$ & Glycerophospholipids & 0.7 & $8.31 \mathrm{E}-18$ & $\downarrow$ \\
\hline 13 & 520.340 & LysoPC (18:2) & C26H50NO7P & $\mathrm{M}+\mathrm{H}$ & HMDB10386 & $\mathrm{C} 04230$ & Glycerophospholipids & 0.5 & $4.50 \mathrm{E}-36$ & $\downarrow$ \\
\hline 14 & 518.323 & LysoPC (18:3) & $\mathrm{C} 26 \mathrm{H} 48 \mathrm{NO} 7 \mathrm{P}$ & $\mathrm{M}+\mathrm{H}$ & HMDB10387 & $\mathrm{C} 04230$ & Glycerophospholipids & 0.5 & $1.75 \mathrm{E}-44$ & $\downarrow$ \\
\hline 15 & 546.352 & LysoPC (20:3) & C28H52NO7P & $\mathrm{M}+\mathrm{H}$ & HMDB10393 & $\mathrm{C} 04230$ & Glycerophospholipids & 0.7 & $6.51 \mathrm{E}-28$ & $\downarrow$ \\
\hline 16 & 544.339 & LysoPC (20:4) & $\mathrm{C} 28 \mathrm{H} 50 \mathrm{NO} 7 \mathrm{P}$ & $\mathrm{M}+\mathrm{H}$ & HMDB10395 & $\mathrm{C} 04230$ & Glycerophospholipids & 0.5 & $3.41 \mathrm{E}-27$ & $\downarrow$ \\
\hline 17 & 502.293 & LysoPE (0:0/20:4) & $\mathrm{C} 25 \mathrm{H} 44 \mathrm{NO} 7 \mathrm{P}$ & $\mathrm{M}+\mathrm{H}$ & HMDB11487 & - & Glycerophospholipids & 0.4 & $1.03 \mathrm{E}-37$ & $\downarrow$ \\
\hline 18 & 828.556 & PC (18:3/22:6) & C48H78NO8P & $\mathrm{M}+\mathrm{H}$ & HMDB08189 & $\mathrm{C} 00157$ & Glycerophospholipids & 0.6 & $1.06 \mathrm{E}-10$ & $\downarrow$ \\
\hline 19 & 780.552 & $\mathrm{PC}(14: 0 / 22: 5)$ & C44H78NO8P & $\mathrm{M}+\mathrm{H}$ & HMDB07890 & $\mathrm{C} 00157$ & Glycerophospholipids & 0.7 & $6.94 \mathrm{E}-15$ & $\downarrow$ \\
\hline 20 & 784.584 & PC (14:1/22:2) & C44H82NO8P & $\mathrm{M}+\mathrm{H}$ & HMDB07921 & $\mathrm{C} 00157$ & Glycerophospholipids & 0.6 & $8.17 \mathrm{E}-32$ & $\downarrow$ \\
\hline 21 & 804.552 & PC $(16: 1 / 22: 6)$ & $\mathrm{C} 46 \mathrm{H} 78 \mathrm{NO} 8 \mathrm{P}$ & $\mathrm{M}+\mathrm{H}$ & HMDB08023 & $\mathrm{C} 00157$ & Glycerophospholipids & 0.5 & $1.15 \mathrm{E}-32$ & $\downarrow$ \\
\hline 22 & 830.567 & $\mathrm{PC}(18: 2 / 22: 6)$ & C48H80NO8P & $\mathrm{M}+\mathrm{H}$ & HMDB08156 & $\mathrm{C} 00157$ & Glycerophospholipids & 0.6 & $4.24 \mathrm{E}-25$ & $\downarrow$ \\
\hline 23 & 782.566 & PC (22:4/14:0) & C44H80NO8P & $\mathrm{M}+\mathrm{H}$ & HMDB08623 & $\mathrm{C} 00157$ & Glycerophospholipids & 0.6 & $1.50 \mathrm{E}-20$ & $\downarrow$ \\
\hline 24 & 766.575 & PC $(16: 1 / 20: 4)$ & C44H80NO7P & $\mathrm{M}+\mathrm{H}$ & HMDB13415 & - & Glycerophospholipids & 0.5 & $3.77 \mathrm{E}-35$ & $\downarrow$ \\
\hline 25 & 819.506 & PG $(18: 3 / 22: 5)$ & $\mathrm{C} 46 \mathrm{H} 75 \mathrm{O} 10 \mathrm{P}$ & $\mathrm{M}+\mathrm{H}$ & HMDB10673 & - & Glycerophospholipids & 40.2 & $1.02 \mathrm{E}-81$ & $\uparrow$ \\
\hline 26 & 807.496 & PI (16:0/16:2) & $\mathrm{C} 41 \mathrm{H} 75 \mathrm{O} 13 \mathrm{P}$ & $\mathrm{M}+\mathrm{H}$ & HMDB09780 & $\mathrm{C} 00626$ & Glycerophospholipids & 23.3 & $5.62 \mathrm{E}-84$ & $\uparrow$ \\
\hline 27 & 838.542 & PE $(22: 5 / 22: 6)$ & $\mathrm{C} 49 \mathrm{H} 76 \mathrm{NO} 8 \mathrm{P}$ & $\mathrm{M}+\mathrm{H}$ & HMDB09639 & $\mathrm{C} 00350$ & Glycerophospholipids & 38.5 & $5.87 \mathrm{E}-80$ & $\uparrow$ \\
\hline 28 & 746.525 & CL $(18: 2 / 18: 1 / 20: 4 / 18: 1)$ & $\mathrm{C} 84 \mathrm{H} 148 \mathrm{O} 17 \mathrm{P} 2$ & $\mathrm{M}+2 \mathrm{H}$ & HMDB58795 & - & Glycerophospholipids & 16.7 & $6.58 \mathrm{E}-67$ & $\uparrow$ \\
\hline 29 & 769.605 & TG (14:1/14:1/18:3) & $\mathrm{C} 49 \mathrm{H} 84 \mathrm{O} 6$ & $\mathrm{M}+\mathrm{H}$ & HMDB47894 & - & Triacylglycerols & 15.5 & $4.00 \mathrm{E}-69$ & $\uparrow$ \\
\hline 30 & 971.594 & TG $(20: 4 / 18: 4 / 18: 4)$ & C59H90O6 & $\mathrm{M}+2 \mathrm{~K}+\mathrm{H}$ & HMDB54281 & - & Triacylglycerols & 41.9 & $5.62 \mathrm{E}-89$ & $\uparrow$ \\
\hline 31 & 371.317 & DG (24:0/0:0/18:2) & $\mathrm{C} 46 \mathrm{H} 86 \mathrm{O} 5$ & $\mathrm{M}+\mathrm{H}+\mathrm{Na}$ & HMDB56120 & - & Glycerolipids & 0.4 & $3.87 \mathrm{E}-39$ & $\downarrow$ \\
\hline 32 & 369.352 & DG $(20: 0 / 24: 0 / 0: 0)$ & $\mathrm{C} 47 \mathrm{H} 92 \mathrm{O} 5$ & $\mathrm{M}+2 \mathrm{H}$ & HMDB07383 & - & Glycerolipids & 0.5 & $5.87 \mathrm{E}-42$ & $\downarrow$ \\
\hline 33 & 305.184 & 19-Hydroxytestosterone & $\mathrm{C} 19 \mathrm{H} 28 \mathrm{O} 3$ & $\mathrm{M}+\mathrm{H}$ & HMDB06769 & C05294 & $\begin{array}{l}\text { Steroids and steroid } \\
\text { derivatives }\end{array}$ & 12.3 & $1.61 \mathrm{E}-63$ & $\uparrow$ \\
\hline 34 & 475.310 & 5b-Cholestane-3a,7a,12a,25-tetrol & $\mathrm{C} 27 \mathrm{H} 48 \mathrm{O} 4$ & $\mathrm{M}+\mathrm{K}$ & HMDB00524 & - & $\begin{array}{c}\text { Steroids and steroid } \\
\text { derivatives }\end{array}$ & 27.3 & $5.70 \mathrm{E}-92$ & $\uparrow$ \\
\hline 35 & 543.293 & Cortolone-3-glucuronide & $\mathrm{C} 27 \mathrm{H} 42 \mathrm{O} 11$ & $\mathrm{M}+\mathrm{H}$ & HMDB10320 & - & $\begin{array}{c}\text { Steroids and steroid } \\
\text { derivatives }\end{array}$ & 18.1 & $1.91 \mathrm{E}-76$ & $\uparrow$ \\
\hline 36 & 586.381 & Estradiol & $\mathrm{C} 18 \mathrm{H} 24 \mathrm{O} 2$ & $2 \mathrm{M}+\mathrm{ACN}+\mathrm{H}$ & HMDB00151 & C00951 & $\begin{array}{l}\text { Steroids and steroid } \\
\text { derivatives }\end{array}$ & 36.9 & $3.86 \mathrm{E}-73$ & $\uparrow$ \\
\hline 37 & 666.137 & NADH & $\mathrm{C} 21 \mathrm{H} 29 \mathrm{~N} 7 \mathrm{O} 14 \mathrm{P} 2$ & $\mathrm{M}+\mathrm{H}$ & HMDB01487 & $\mathrm{C} 00004$ & $\left(5^{\prime}->5^{\prime}\right)$-dinucleotides & 5.4 & $3.46 \mathrm{E}-43$ & $\uparrow$ \\
\hline 38 & 393.298 & Chenodeoxycholic acid & $\mathrm{C} 24 \mathrm{H} 40 \mathrm{O} 4$ & $\mathrm{M}+\mathrm{H}$ & HMDB00518 & $\mathrm{C} 02528$ & $\begin{array}{l}\text { Bile acids, alcohols } \\
\text { and derivatives }\end{array}$ & 0.5 & $1.39 \mathrm{E}-33$ & $\downarrow$ \\
\hline 39 & 627.109 & Pyrogallol-2-O-glucuronide & $\mathrm{C} 12 \mathrm{H} 14 \mathrm{O} 9$ & $2 \mathrm{M}+\mathrm{Na}$ & HMDB60017 & - & $\begin{array}{l}\text { Carbohydrates } \\
\text { and carbohydrate } \\
\text { conjugates }\end{array}$ & 4.5 & $1.34 \mathrm{E}-41$ & $\uparrow$ \\
\hline 40 & 685.842 & Foetidissimoside B & C63H100O31 & $\mathrm{M}+\mathrm{H}+\mathrm{NH} 4$ & HMDB36252 & - & Prenol Lipids & 2.8 & $7.43 \mathrm{E}-21$ & $\uparrow$ \\
\hline
\end{tabular}




\begin{tabular}{|c|c|c|c|c|c|c|c|c|c|c|}
\hline No. & $\mathbf{m} / \mathbf{z}$ & Identify Result & $\begin{array}{c}\text { Element } \\
\text { composing }\end{array}$ & Ion & HMDB ID & KEGG ID & Class & Fold Change & p-value & Trend \\
\hline 41 & 315.080 & Geranyl-PP & $\mathrm{C} 10 \mathrm{H} 20 \mathrm{O} 7 \mathrm{P} 2$ & $\mathrm{M}+\mathrm{H}$ & HMDB01285 & $\mathrm{C} 00341$ & Prenol Lipids & 0.0 & $2.38 \mathrm{E}-32$ & $\downarrow$ \\
\hline 42 & 283.201 & Vitamin A2 aldehyde & $\mathrm{C} 20 \mathrm{H} 26 \mathrm{O}$ & $\mathrm{M}+\mathrm{H}$ & HMDB35695 & C05918 & Prenol lipids & 19.9 & 4.61E-78 & $\uparrow$ \\
\hline 43 & 409.161 & Melatonin glucuronide & $\mathrm{C} 19 \mathrm{H} 24 \mathrm{~N} 2 \mathrm{O} 8$ & $\mathrm{M}+\mathrm{H}$ & HMDB60830 & - & $\begin{array}{c}\text { Nucleoside and } \\
\text { nucleotide analogues }\end{array}$ & 0.6 & $2.62 \mathrm{E}-24$ & $\downarrow$ \\
\hline 44 & 525.304 & VPGPR Enterostatin & $\mathrm{C} 23 \mathrm{H} 40 \mathrm{~N} 8 \mathrm{O} 6$ & $\mathrm{M}+\mathrm{H}$ & HMDB03577 & - & $\begin{array}{c}\text { Carboxylic acids and } \\
\text { derivatives }\end{array}$ & 12.4 & $4.26 \mathrm{E}-69$ & $\uparrow$ \\
\hline
\end{tabular}

44 significant difference metabolites were screened out to be the potential biomarkers according to the value of variable importance for projection (VIP) value in PLS-DA model, the fold changes and P-value between the PV and HC samples were calculated. These potential biomarkers were involved in glycerophospholipid metabolism, sphingolipid metabolism, arachidonic acid metabolism, bile acid biosynthesis.

a Trend change compared with healthy controls (HC). $(\uparrow)$ and $(\downarrow)$ represent the corresponding compound is up- regulated and down-regulated in PV patients compared with the HC, respectively.

LysoPC: Lysophosphatidylcholine

LysoPE: Lysophosphatidyl ethanolamine

PC: Phosphatidylcholine

PG: Phosphatidylglycerol

PI: Phosphatidylinositol

PE: Phosphatidylethanolamine

CL: Cardiolipin

TG: Tracylglycerol

DG: Diacylglycerol

NADH: Nicotinamide adenine dinucleotide

VPGPR: Val-Pro-Gly-Pro-Arg

Table 2: Clinical characteristics of psoriasis patients and healthy controls

\begin{tabular}{lcc}
\hline Characteristics & PV Patients & Healthy Controls \\
\hline Number of subjects & 75 & 75 \\
Gender (male/female) & $50 / 25$ & $47 / 28$ \\
Age & $36.7 \pm 13.1$ & $33.3 \pm 12.2$ \\
PASI score & & \\
mild (PASI score $\leq 10)$ & 37 & - \\
moderate to severe (PASI score $>10)$ & 38 & - \\
\hline
\end{tabular}

The clinical characteristics of 75 psoriasis vulgaris patients and 75 healthy volunteers were described.

pathways. The visualized result facilitated further biological interpretation to reveal the most relevant pathways as shown in Figure 6. The different color and size of the symbol means the different level of significance, there were more potential biomarkers in the data were involved in the pathway, the color was darker or the size was larger. As Figure 6 shown, the glycerophospholipid metabolism, sphingolipid metabolism, arachidonic acid metabolism was most relevant pathways in psoriasis metabolism.

\section{Correlation network}

To investigate the latent relationships of the 44 metabolites, the Pearson correlation coefficients between the metabolites were calculated on the basis of the average normalized quantities of metabolites. Highly correlated metabolites with $r>0.8$ were connected with solid lines, which metabolite with $\mathrm{r}<-0.8$ were connected with dotted lines in the network (Figure 7). Orange plots indicated up-regulated metabolites and green plots indicated downregulated metabolites in $\mathrm{PV}$.

Most metabolites in lysophospholipid and phospholipid were down-regulated in PV groups and had highly correlated coefficients. Levels of some correlated arachidonic acid, inflammatory factor, sphingolipid were up-regulated.

\section{Receiver operating characteristic curve}

ROC curve analysis is generally considered to be the gold standard for the assessment of biomarker performance. The results of ROC curve analysis of the 44 differentiated metabolites guaranteed the reliability of potential biomarkers for wide and qualified independent validation. The value of area under the curve (AUC) was higher than 0.7 suggested good predict ability of potential biomarkers. Typical ROC curves and AUC values were shown in Figure 8A $\sim$ E. Multivariate exploratory ROC analysis overview was shown in Figure $8 \mathrm{~F}$.

\section{DISCUSSION}

We developed an untargeted metabonomics method to investigate the pathogenesis of psoriasis, 44 potential biomarkers were screened out, there involved in several metabolic pathways. 


\section{Arachidonic acid metabolism}

Leukotrienes are a family of eicosanoid inflammatory mediators produced in leukocytes by the oxidation of arachidonic acid by the enzyme arachidonate 5-lipoxygenase. Leukotrienes can regulate immune responses by using lipid signaling to convey information to either the cell producing them (autocrine signaling) or neighboring cells (paracrine signaling). The product of Leukotriene such as histamine and prostaglandins are also the inflammatory mediators.

Leukotriene D4 (LTD4) is a potent inflammatory mediator. It is also a pro-inflammatory mediator present at high levels in many inflammatory diseases, caused increasing risk for subsequent cancer development in chronic inflammation. LTD4 have been mentioned to associate with the pathogenesis of several inflammatory disorders, such as asthma and inflammatory bowel disease.

Leukotriene E3 is an eicosanoid derived from 8,11,14-eicosatrienoic acid by the 5-lipoxygenaseleukotriene pathway. Leukotriene $\mathrm{E} 3$ is also a by-product of the metabolism of leukotriene $\mathrm{C} 3$. The eicosanoids are a diverse family of molecules consists of the prostaglandins (PGs), thromboxanes (TXs), leukotrienes (LTs) and lipoxins (LXs) have powerful effects on cell function. The eicosanoids had been known that they were involved in asthma, pain, fever and vascular responses, present evidence indicates that eicosanoids exert relevant effects on immune, as well as structural, cells pertinent to fibrogenesis [39-42].

Our result revealed that the content of Leukotriene D4 and Leukotriene E3 in serum was significantly higher in psoriasis patients than that in healthy controls. Arachidonic acid metabolism abnormality was an important factor in pathogenesis of psoriasis. This result is consistent with our previous studies on urine metabolome of psoriasis patients [37].

\section{Lipid metabolism}

In this study, some lipid compounds were significant different between PV and HC, involved in glycerophospholipid metabolism, Phospholipid Biosynthesis and sphingolipid metabolism.
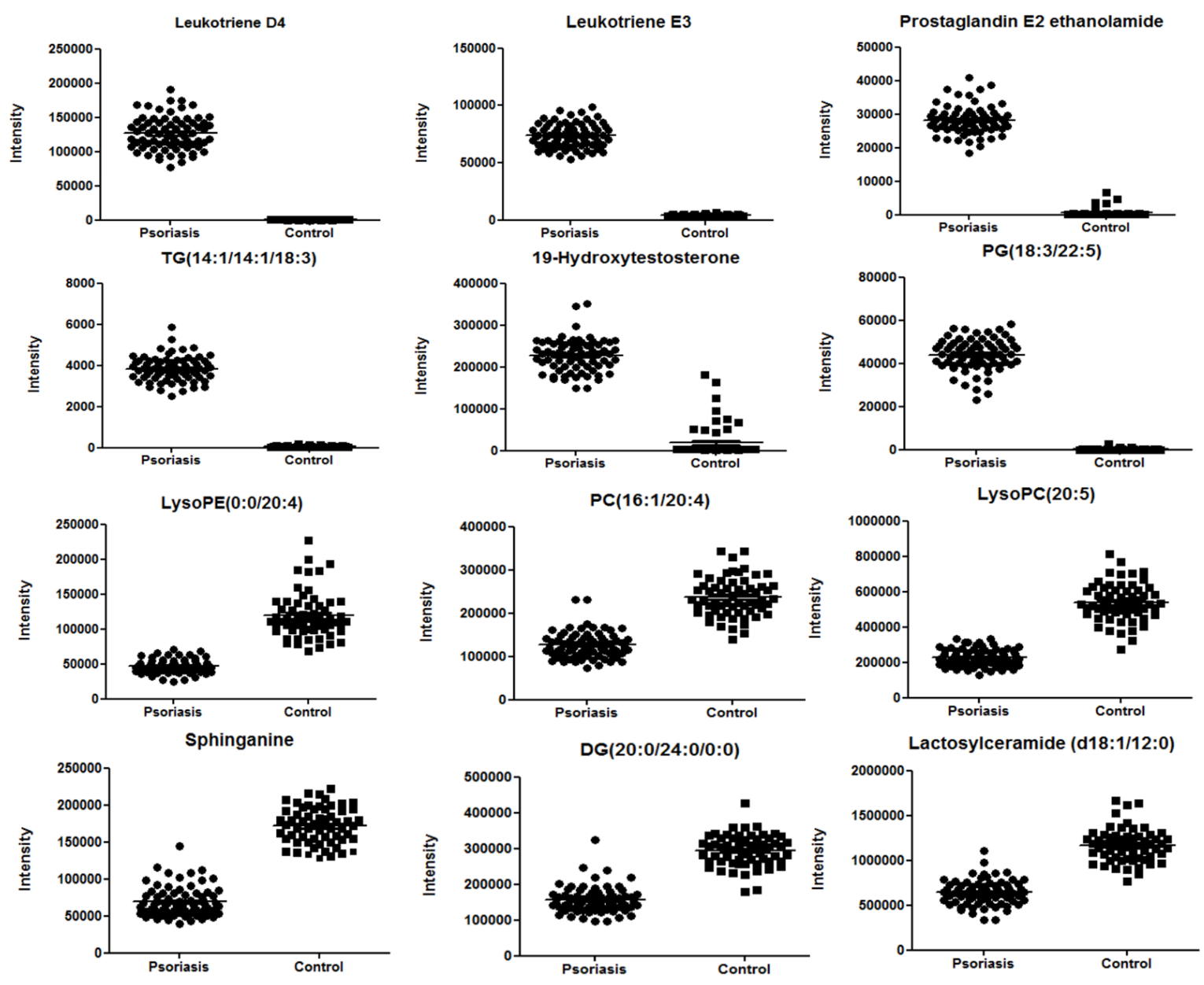

Figure 4: Typical metabolites with significant alterations in psoriasis vs. control. The peaks intensities of potential biomarkers in serum of the two groups were shown. 
In recent years, more and more lipid metabolism disorders in psoriasis have been reported. There was a significant relationship between severity of psoriasis and serum lipid profile. This may elevate the risk of atherosclerosis, particularly cardiovascular disorders [43, 44]. Epidemiological studies and clinical investigation research showed patients had high prevalence of cardiovascular comorbidities, and high cardiovascular risk according to the Framingham risk score [45-47].

Sphingolipids have important biological and structural functions in the maintenance of the skin barrier function and regulate cellular processes including proliferation, differentiation and apoptosis of keratinocytes. Previous studies results showed that the composition change and metabolism disorder of epidermal sphingolipids were happened in many dermatologic diseases such as psoriasis, atopic dermatitis and ichthyoses [48].

The prostanoids and leukotrienes as the lipid mediators are metabolites of arachidonic acid mediated through their respective receptors expressed on target cells and released in various pathophysiological conditions. It has been proved that the balance of the production and the receptor expression of each lipid mediator are important for the homeostasis maintaining in our body. The functions of prostanoids and leukotrienes in skin inflammatory diseases focusing on contact dermatitis, atopic dermatitis, and psoriasis had been reviewed [49-51].

Therefore, the lipid compounds may to be the diagnostic and examination index in psoriasis in future.

\section{Bile acid biosynthesis}

Chenodeoxycholic acid is a bile acid which can facilitate excretion, absorption, and transport of fats and sterols in the intestine and liver, usually considered to be a physiological detergent. Bile acids are also the steroidal amphipathic molecules derived from the catabolism of cholesterol. They are essential for the absorption of dietary fats and vitamins, participate in the regulation of all the key enzymes involved in cholesterol homeostasis, and also modulate bile flow and lipid secretion. The unique detergent properties of bile acids are essential for the digestion and intestinal absorption of hydrophobic nutrients [52-55]. In this study, the content of chenodeoxycholic acid in PV was lower than HC. The

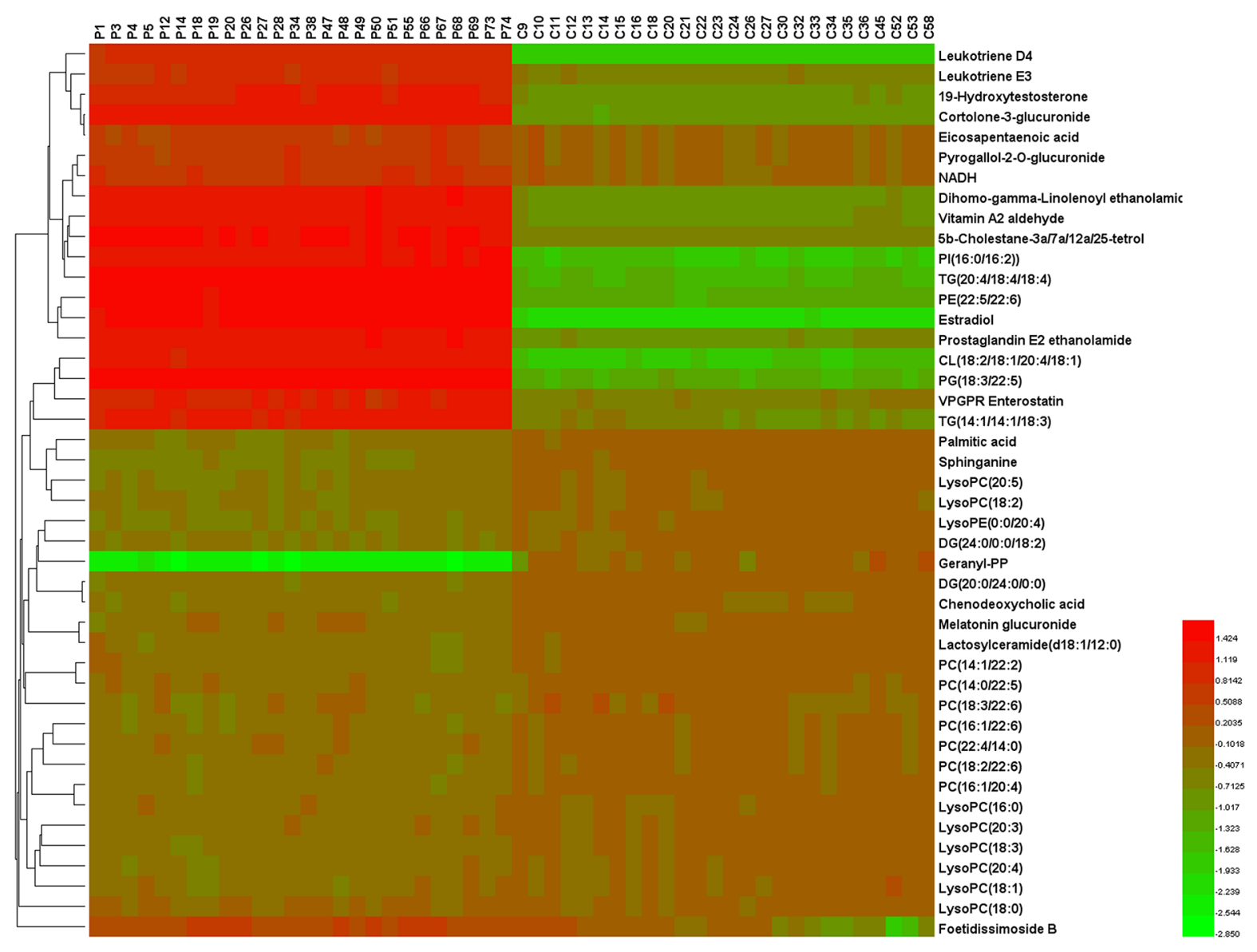

Figure 5: Heat map of the 44 differential metabolites. (Psoriasis patients are labelled P... and healthy controls are labelled C...). The ratio of metabolite in the subject samples to the average of those in the healthy control samples was calculated, and then the metabolic alteration was demonstrated as $\log 10$ (ratio), the major metabolic alterations in psoriasis were visualized in a heat map plot. 


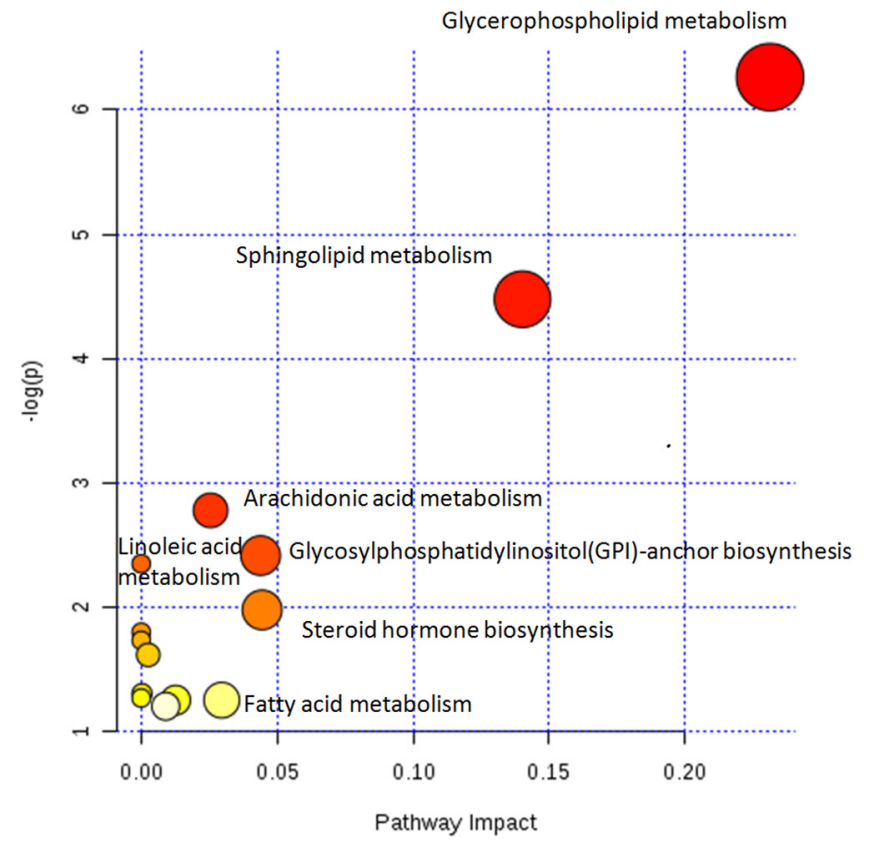

Figure 6: Disturbed metabolic pathways showed various metabolism changes when comparing psoriasis and control. The metabolic pathway analysis was an integrating enrichment analysis based on the human metabolic pathways. The different color and size of the symbol means the different level of significance, there were more potential biomarkers in the data were involved in the pathway, the color was darker or the size was larger. Glycerophospholipid metabolism, sphingolipid metabolism, arachidonic acid metabolism was most relevant pathways in psoriasis metabolism.

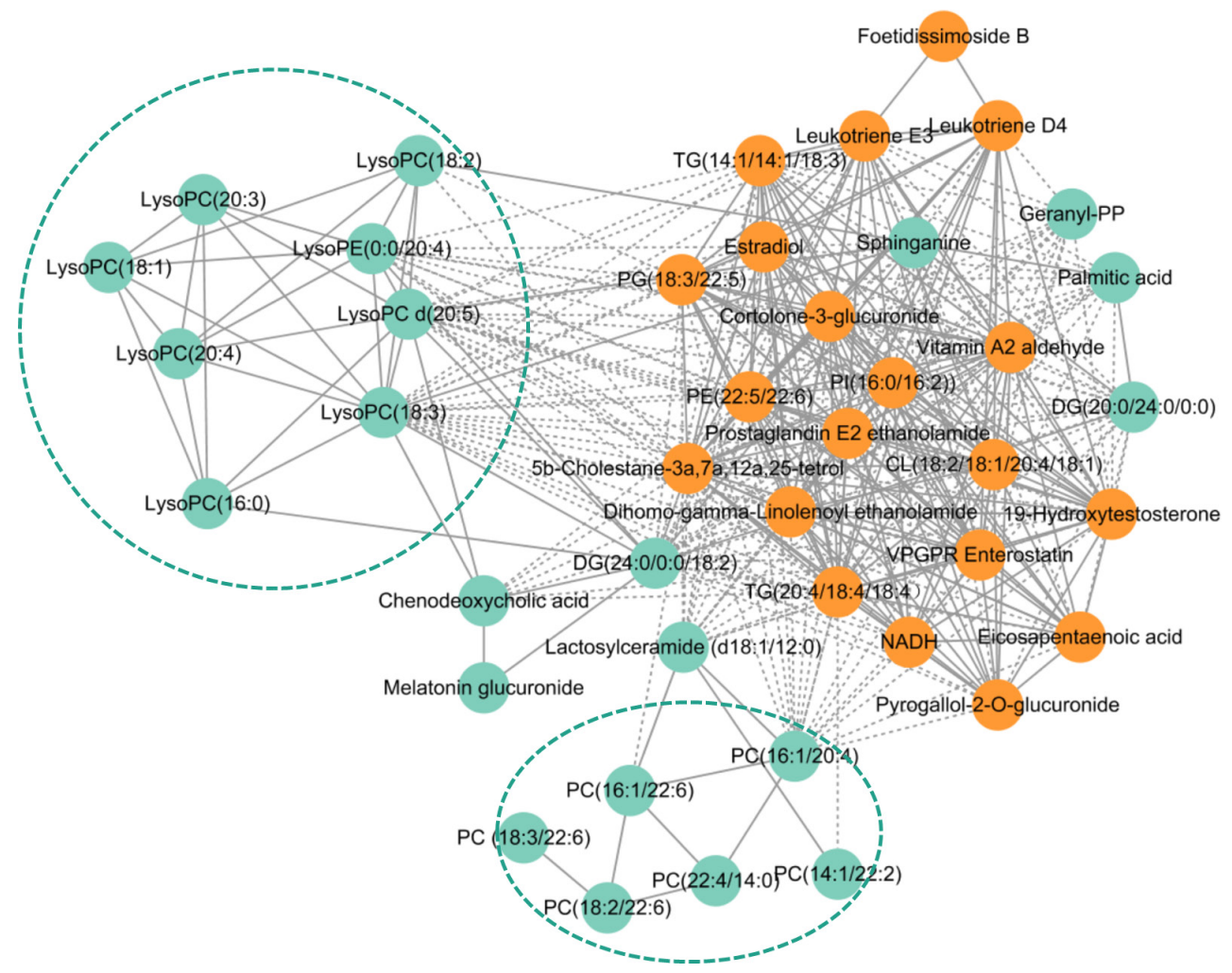

Figure 7: Pearson correlation network of metabolites. Pearson correlation coefficients between the 44 metabolites were calculated on the basis of the average normalized quantities of metabolites. Highly correlated metabolites with $r>0.8$ were connected with solid lines, which metabolite with $\mathrm{r}<-0.8$ were connected with dotted lines in the network. Orange plots indicated up-regulated metabolites and green plots indicated down-regulated metabolites in PV. 
deficiency of bile acids might involve in the pathogenesis of psoriasis [56, 57].

In this study, an untargeted high-throughput liquid chromatography coupled to mass spectrometry method was carried out for the global metabonomics analysis with the purpose of finding out more potential biomarkers in human serum for diagnosing psoriasis vulgaris patients and exploring pathogenesis of psoriasis. The levels of the 44 potential biomarkers demonstrated there were significant differences between $\mathrm{PV}$ patients and $\mathrm{HC}$, the psoriasis patients groups with different severity also showed the different metabolic trajectory. These 44 potential biomarkers provided a better comprehension of the pathophysiological progress of PV, indicated the psoriasis may involve in several disturbed metabolic pathways including glycerophospholipid metabolism, sphingolipid metabolism, arachidonic acid metabolism, bile acid biosynthesis. Furthermore, this study was an exploratory research, these potential biomarkers still need repeated verified by more clinical samples and quantitative analysis by standards. To illustrate the pathogenesis of psoriasis systematically and comprehensively, the metabonomics results should be integrated and compared with the outcomes of other 'omics' studies such as transcriptomics, proteomics and genomics, it will to be benefit to discover the biomarkers group, and apply them to real clinical disease diagnostics and treatments.
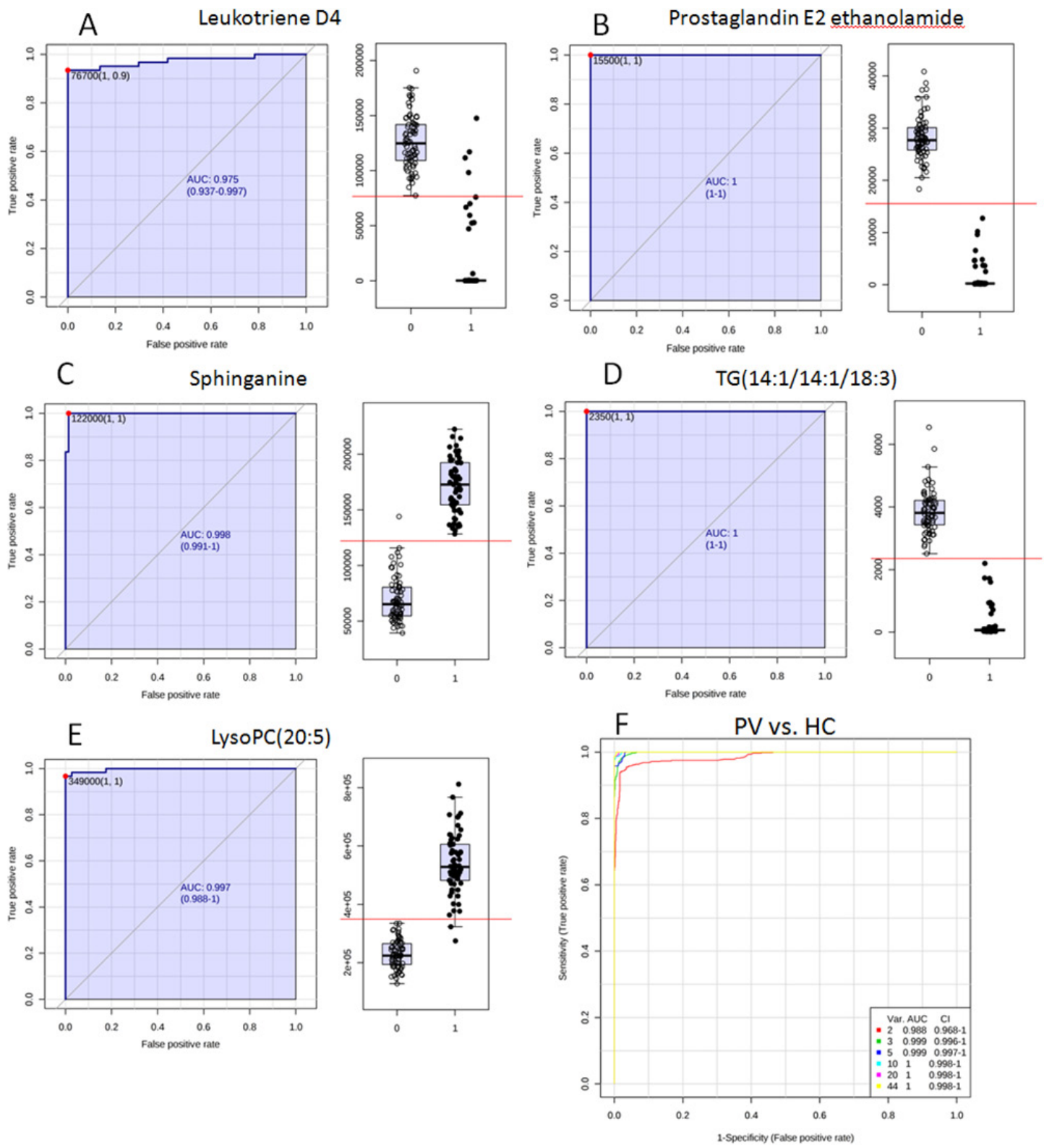

Figure 8: The typical ROC curve plots of potential biomarkers. (A E) Typical ROC curves and AUC values of potential biomarkers with high-performance prediction; (F) Multivariate exploratory ROC analysis overview. 


\section{MATERIALS AND METHODS}

\section{Human serum collection}

75 out patients confirmed psoriasis vulgaris were recruited from the dermatology department of Guangdong provincial hospital of Chinese medicine (Guangzhou, Guangdong, China) and dermatology department of people's liberation army 195 hospital (Xianning, Hubei, China). 75 healthy volunteers were recruited from physical examination department of Guangdong provincial hospital of Chinese medicine. Clinical characteristics of patient and controls were described as Table 2 . The study protocol (No. B2010-08-01) was approved by the institutional ethics committee of Guangdong provincial hospital of Chinese medicine, and all of the patients in this study have understood and signed informed consent.

All individuals were asked to be on empty stomach for 8-14 hours, and refrained from drinking, smoking for 24 hours before serum collection. Serum was prepared by evacuated and promoting coagulating tubes and centrifugation (3000 rpm, 10min). The upper serum layer was stored at $-80^{\circ} \mathrm{C}$ until sample analysis.

\section{Reagents and instrumentations}

Acetonitrile (HPLC grade) was purchased from Merck (Darmstadt, Germany). Formic acid (analytical grade) was purchased from Fluka (Buchs, Switzerland). Ultrapure water (18.2 M $\Omega$ ) was prepared by using a Milli-Q water purification system (Millipore, Bedford, MA, USA).

Serum metabolic profiling data were acquired by an ultra-performance liquid chromatography (Waters ACQUITY UPLC, USA) coupled with a quadruple-timeof-flight mass spectrometry (AB SCIEX Triple-TOF 5600 , USA). Vortex mixer was purchased from Qilinbeier (VORTEX-5, Jiangsu, China), Centrifuge was purchased from Beckman Coulter (AllegraTM X-22, Beckman Coulter Corporation, USA).

\section{Sample preparation}

The frozen serum samples thawed at $4^{\circ} \mathrm{C}, 100 \mu \mathrm{L}$ serum was mixed with $400 \mu \mathrm{L}$ acetonitrile and vortexed for $2 \mathrm{~min}$, then centrifuged at $13,000 \mathrm{rpm}$ for $20 \mathrm{~min}$ at $4^{\circ} \mathrm{C}$. The supernatant was collected and transferred to a 96 well collection plate waiting for LC-MS analysis.

\section{LC-MS analysis}

Chromatography was carried out by an ACQUITY BEH C18 chromatography column $(100 \times 2.1 \mathrm{~mm}, 1.7 \mu \mathrm{m}$, Waters). The mobile phases consisted of ultra-pure water with $0.1 \%$ formic acid (phase A) and acetonitrile with $0.1 \%$ formic acid (phase B). A linear gradient elution was carried out. At first, $2 \%$ mobile phase B was run for $1 \mathrm{~min}$, then quickly increased to $15 \%$ in $1 \mathrm{~min}$ and kept for $5 \mathrm{~min}$, followed by a linear increase to $65 \%$ mobile phase B from 7 to $15 \mathrm{~min}$, and then further increased to $95 \%$ mobile phase B from 15 to $23 \mathrm{~min}$, kept $95 \%$ for $8 \mathrm{~min}$, at last the proportion of phase B returned to $2 \%$ in $0.1 \mathrm{~min}$, and the column was allowed to re-equilibrate for $2 \mathrm{~min}$ before the next injection. The column temperature was maintained at $40^{\circ} \mathrm{C}$. The flow rate was $0.4 \mathrm{~mL} / \mathrm{min}$. The temperature of auto-sampler was maintained at a $4^{\circ} \mathrm{C}$ for the duration of analysis, and the sample injection volume was $2 \mu \mathrm{L}$.

The data were acquired from m/z 100 to 1000 in positive electrospray ionization $(\mathrm{ESI}+)$ mode. The ion source voltage flow (ISVF) was set to 5500, source temperature was set to $650^{\circ} \mathrm{C}$. The information dependent acquisition (IDA) function was enabled to obtain the high resolution, high mass accuracy MS/MS spectra. All the acquired $\mathrm{m} / \mathrm{z}$ data were real-time corrected using an independent reference to ensure the higher accuracy.

\section{Raw data processing and statistical analysis}

The raw spectrometric data were collected by Analyst ${ }^{\circledR}$ TF Software Kit (Version 1.5.1, AB SCIEX, USA), and processed by the MarkerView ${ }^{\mathrm{TM}}$ software (Version 1.2.1, AB SCIEX, USA) for chromatography peak recognition and matching, noise filtering, data normalization, peak list data were loaded to Simca-P software (version 14.1, Umetrics, Umeå, Sweden) for multivariate data analysis. Principal components analysis (PCA), partial least squares discriminate analysis (PLSDA) and orthogonal signal correction partial least squares discriminate analysis (OPLS-DA) was applied to distinguish psoriasis vulgaris (PV) patients from healthy controls (HC). The metabolites with the variable importance in the projection (VIP) values $>1$ in the PLS-DA model were selected as candidate potential biomarkers. The P-value cut-off in t-test was set to 0.05 [58]. Furthermore, the generalization ability of fitting models was evaluated by 200 permutations of cross-test validation. As the LC-MS analysis provided the retention time, precise molecular mass and MS/MS information of the metabolites, potential biomarkers could be identified. We screened out the candidate by PeakView ${ }^{\circledR}$ Software (Version 1.2, AB SCIEX, USA), current free metabolite databases such as The Human Metabolome Database (HMDB, http://www.hmdb.ca/), KEGG (http://www. genome.jp/kegg) were used to search for molecule formula and structures [59-61]. A heat map was plotted to illustrate up-regulations and down-regulations in significant change metabolites by using Heml 1.0 (Heat map illustrator). A correlation network was plotted by Cytoscape software package version 3.2.1 (National Institute of General Medical Sciences, Bethesda, MD, USA). MetaboAnalyst 3.0 (www.metaboanalyst.ca) was applied to draw the receiver operating characteristic (ROC) curves of the potential biomarkers [62]. MetaboAnalyst 3.0 was 
also applied to analyze pathways for discovering the most important metabolic pathway which significant metabolites enriched in.

\section{ACKNOWLEDGMENTS AND FUNDING}

This work was supported by grants from the natural science foundation of China (81302736), the Guangdong natural science foundation research team project (S2013030011515), the Guangdong natural science foundation (S2011040005849), the post-doctor science foundation (2012M510196), the Guangzhou university of Chinese medicine innovation foundation (2011KT1842), and the Guangdong education foundation (2014KT1386).

Thanks for the cooperation of People's Liberation Army 195 Hospital, Xianning, Hubei, China.

\section{CONFLICTS OF INTEREST}

The authors declare no conflicts of interest.

\section{REFERENCES}

1. Parisi R, Symmons DPM, Griffiths CEM, Ashcroft DM, Identification M. Global Epidemiology of Psoriasis: A Systematic Review of Incidence and Prevalence. Journal of Investigative Dermatology. 2013; 133:377-85. https://doi. org/10.1038/jid.2012.339.

2. Christophers E. Psoriasis - epidemiology and clinical spectrum. Clinical and Experimental Dermatology. 2001; 26:314-20. https://doi.org/10.1046/j.1365-2230.2001.00832.x.

3. Nevitt GJ, Hutchinson PE. Psoriasis in the community: Prevalence, severity and patients' beliefs and attitudes towards the disease. British Journal of Dermatology. 1996; 135:533-7. https://doi.org/10.1111/j.1365-2133.1996. tb03826.x

4. Pompili M, Innamorati M, Trovarelli S, Narcisi A, Bellini S, Orsini D, Forte A, Erbuto D, Botti E, Lamis DA, Girardi P, Costanzo A. Suicide risk and psychiatric comorbidity in patients with psoriasis. Journal of International Medical Research. 2016; 44:61-6. https://doi. org $/ 10.1177 / 0300060515593253$.

5. Machado-Pinto J, Diniz MdS, Bavoso NC. Psoriasis: new comorbidities. Anais Brasileiros De Dermatologia. 2016; 91:8-14. https://doi.org/10.1590/abd1806-4841.20164169.

6. Cohen AD, Sherf M, Vidavsky L, Vardy DA, Shapiro J, Meyerovitch J. Association between psoriasis and the metabolic syndrome. A cross-sectional study. Dermatology (Basel, Switzerland). 2008; 216:152-5. https://doi. org/10.1159/000111512.

7. Takahashi H, Iizuka H. Psoriasis and metabolic syndrome. Journal of Dermatology. 2012; 39:212-8. https://doi. org/10.1111/j.1346-8138.2011.01408.x.
8. Armstrong AW, Harskamp CT, Armstrong EJ. Psoriasis and metabolic syndrome: A systematic review and meta-analysis of observational studies. Journal of the American Academy of Dermatology. 2013; 68:654-62. https://doi.org/10.1016/j. jaad.2012.08.015.

9. Salihbegovic EM, Hadzigrahic N, Cickusic AJ. Psoriasis and metabolic syndrome. Medical archives (Sarajevo, Bosnia and Herzegovina). 2015; 69:85-7. https://doi. org/10.5455/medarh.2015.69.85-87.

10. Szponar-Bojda A, Krasowska D, Pietrzak A, Chodorowska G. Metabolic syndrome in psoriasis. Postepy Dermatologii I Alergologii. 2012; 29:356-62. https://doi.org/10.5114/ pdia.2012.31488.

11. Alsufyani MA, Golant AK, Lebwohl M. Psoriasis and the metabolic syndrome. Dermatologic Therapy. 2010; 23:137-43.

12. Lowes MA, Kikuchi T, Fuentes-Duculan J, Cardinale I, Zaba LC, Haider AS, Bowman EP, Krueger JG. Psoriasis vulgaris lesions contain discrete populations of Th1 and Th17 T cells. Journal of Investigative Dermatology. 2008; 128:1207-11. https://doi.org/10.1038/sj.jid.5701213.

13. Zhang XJ, Huang W, Yang S, Sun LD, Zhang FY, Zhu QX, Zhang FR, Zhang C, Du WH, Pu XM, Li H, Xiao FL, Wang ZX, et al. Psoriasis genome-wide association study identifies susceptibility variants within LCE gene cluster at 1q21. Nature Genetics. 2009; 41:205-10. https://doi. org/10.1038/ng.310.

14. Adebajo A, Boehncke WH, Gladman DD, Mease PJ. Psoriatic Arthritis and Psoriasis. Springer International Publishing. 2016.

15. Nestle FO, Kaplan DH, Barker J. Mechanisms of Disease: Psoriasis. New England Journal of Medicine. 2009; 361:496-509. https://doi.org/10.1056/NEJMra0804595.

16. Thorslund K, Dufva AE, Amatya B, Nordlind K. Expression of the serotonin transporter protein in psoriasis is correlated with the severity of the disease and chronic stress. Journal of the American Academy of Dermatology. 2013; 68:AB3-AB.

17. Wang $H$, von Rohrscheidt J, Roehrbein J, Peters $T$, Sindrilaru A, Kess D, Preissner KT, Scharffetter-Kochanek K. Extracellular Adherence Protein of Staphylococcus aureus Suppresses Disease by Inhibiting T-Cell Recruitment in a Mouse Model of Psoriasis. Journal of Investigative Dermatology. 2010; 130:743-54. https://doi.org/10.1038/ jid.2009.310.

18. Coimbra S, Oliveira H, Figueiredo A, Rocha-Pereira P, Santos-Silva A. Psoriasis: Epidemiology, Clinical and Histological Features, Triggering Factors, Assessment of Severity and Psychosocial Aspects. InTech. 2012; 3. https:// doi.org/10.5772/26474

19. Toker A, Kadi M, Yildirim AK, Aksoy H, Akcay F. Serum lipid profile paraoxonase and arylesterase activities in psoriasis. Cell Biochemistry and Function. 2009; 27:17680. https://doi.org/10.1002/cbf.1553. 
20. Mallbris L, Granath F, Hamsten A, Stahle M. Psoriasis is associated with lipid abnormalities at the onset of skin disease. Journal of the American Academy of Dermatology. 2006; 54:614-21. https://doi.org/10.1016/j. jaad.2005.11.1079.

21. Pietrzak A, Chodorowska G, Szepietowski J, ZalewskaJanowska A, Krasowska D, Hercogova J. Psoriasis and serum lipid abnormalities. Dermatologic Therapy. 2010; 23:160-73.

22. Colsman A, Brugel M, Thiery J, Sticherling M. Lipid metabolism and psoriasis vulgaris. Journal of the European Academy of Dermatology and Venereology. 2007; 21:27.

23. Murzaku EC, Bronsnick T, Rao BK. Diet in dermatology: Part II. Melanoma, chronic urticaria, and psoriasis. Journal of the American Academy of Dermatology. 2014; 71:1053. e1-.e16. https://doi.org/10.1016/j.jaad.2014.06.016.

24. Jiang S, Hinchliffe TE, Wu T. Biomarkers of An Autoimmune Skin Disease-Psoriasis. Genomics Proteomics \& Bioinformatics. 2015; 13:224-33. https://doi. org/10.1016/j.gpb.2015.04.002.

25. Takahashi H, Takahashi I, Honma M, Ishida-Yamamoto A, Iizuka H. Prevalence of metabolic syndrome in Japanese psoriasis patients. Journal of Dermatological Science. 2010; 57:143-4. https://doi.org/10.1016/j.jdermsci.2009.11.002.

26. Gisondi P, Tessari G, Conti A, Piaserico S, Schianchi S, Peserico A, Giannetti A, Girolomoni G. Prevalence of metabolic syndrome in patients with psoriasis: a hospital-based case-control study. British Journal of Dermatology. 2007; 157:68-73. https://doi. org/10.1111/j.1365-2133.2007.07986.x.

27. Armstrong AW, Wu J, Johnson MA, Grapov D, Azizi B, Dhillon J, Fiehn O. Metabolomics in psoriatic disease: pilot study reveals metabolite differences in psoriasis and psoriatic arthritis. F1000Research. 2014; 3:248. https://doi. org/10.12688/f1000research.4709.1.

28. Kamleh MA, Snowden SG, Grapov D, Blackburn GJ, Watson DG, Xu N, Stahle M, Wheelock CE. LC-MS Metabolomics of Psoriasis Patients Reveals Disease Severity-Dependent Increases in Circulating Amino Acids That Are Ameliorated by Anti-TNF alpha Treatment. Journal of Proteome Research. 2015; 14:557-66. https:// doi.org/10.1021/pr500782g.

29. Kang H, Li X, Zhou Q, Quan C, Xue F, Zheng J, Yu Y. Exploration of candidate biomarkers for human psoriasis based on gas chromatography-mass spectrometry serum metabolomics. British Journal of Dermatology. 2017; 176:713-22. https://doi.org/10.1111/bjd.15008.

30. Brindle JT, Antti H, Holmes E, Tranter G, Nicholson JK, Bethell HWL, Clarke S, Schofield PM, McKilligin E, Mosedale DE, Grainger DJ. Rapid and noninvasive diagnosis of the presence and severity of coronary heart disease using H-1-NMR-based metabonomics. Nature Medicine. 2002; 8:1439-44. https://doi.org/10.1038/nm802.
31. Nicholson JK, Lindon JC, Holmes E. 'Metabonomics': understanding the metabolic responses of living systems to pathophysiological stimuli via multivariate statistical analysis of biological NMR spectroscopic data. Xenobiotica. 1999; 29:1181-9.

32. Sreekumar A, Poisson LM, Rajendiran TM, Khan AP, Cao Q, Yu J, Laxman B, Mehra R, Lonigro RJ, Li Y, Nyati MK, Ahsan A, Kalyana-Sundaram S, et al. Metabolomic profiles delineate potential role for sarcosine in prostate cancer progression. Nature. 2009; 457:910-4. https://doi. org/10.1038/nature07762.

33. Dumas ME, Kinross J, Nicholson JK. Metabolic Phenotyping and Systems Biology Approaches to Understanding Metabolic Syndrome and Fatty Liver Disease. Gastroenterology. 2014; 146:46-62. https://doi. org/10.1053/j.gastro.2013.11.001.

34. Tian Y, Nie X, Xu S, Li Y, Huang T, Tang HR, Wang YL. Integrative metabonomics as potential method for diagnosis of thyroid malignancy. Scientific Reports. 2015; 5:12. https://doi.org/10.1038/srep14869.

35. Ou ZJ, Li L, Liao XL, Wang YM, Hu XX, Zhang QL, Wang ZP, Yu H, Zhang X, Hu P, Xu YQ, Liang QL, Ou JS, et al. Apolipoprotein A-I mimetic peptide inhibits atherosclerosis by altering plasma metabolites in hypercholesterolemia. American Journal of Physiology-Endocrinology and Metabolism. 2012; 303:E683-E94. https://doi.org/10.1152/ ajpendo.00136.2012.

36. Gao X, Guo M, Li Q, Peng L, Liu H, Zhang L, Bai X, Wang Y, Li J, Cai C. Plasma Metabolomic Profiling to Reveal Antipyretic Mechanism of Shuang-Huang-Lian Injection on Yeast-Induced Pyrexia Rats. Plos One. 2014; 9. https:// doi.org/10.1371/journal.pone.0100017.

37. Lu C, Deng J, Li L, Wang D, Li G. Application of metabolomics on diagnosis and treatment of patients with psoriasis in traditional Chinese medicine. Biochimica Et Biophysica Acta-Proteins and Proteomics. 2014; 1844:2808. https://doi.org/10.1016/j.bbapap.2013.05.019.

38. Xie YY, Li L, Shao Q, Wang YM, Liang QL, Zhang HY, Sun P, Qiao MQ, Luo GA. Urinary metabolomics study on an induced-stress rat model using UPLC-QTOF/MS. Rsc Advances. 2015; 5:75111-20. https://doi.org/10.1039/ c5ra10992b.

39. Charbeneau RP, Peters-Golden M. Eicosanoids: mediators and therapeutic targets in fibrotic lung disease. Clinical Science. 2005; 108:479-91.

40. Luo M, Flamand N, Brock TG. Metabolism of arachidonic acid to eicosanoids within the nucleus. Biochimica Et Biophysica Acta-Molecular and Cell Biology of Lipids. 2006; 1761:618-25. https://doi.org/10.1016/j. bbalip.2006.02.018.

41. Loick HM, Theissen JL. Eicosanoids as mediators in ARDS. Anasthesiologie, Intensivmedizin, Notfallmedizin, Schmerztherapie: AINS. 1994; 29:3-9. 
42. Hammarstrom S, Bernstrom K, Orning L, Dahlen SE, Hedqvist P. Rapid in vivo metabolism of leukotriene C3 in the monkey Macaca irus. Biochemical and biophysical research communications. 1981; 101:1109-15. https://doi. org/10.1016/0006-291x(81)91562-x.

43. Xia J, Wishart DS. Using MetaboAnalyst 3.0 for Comprehensive Metabolomics Data Analysis. Current protocols in bioinformatics. 2016; 55:14.0.1-.0.91. https:// doi.org/10.1002/cpbi.11.

44. Taheri Sarvtin M, Hedayati MT, Shokohi T, HajHeydari Z. Serum lipids and lipoproteins in patients with psoriasis. Archives of Iranian medicine. 2014; 17:343-6. https://doi. org/0141705/aim.007.

45. Owczarczyk-Saczonek AB, Nowicki RJ. Prevalence of cardiovascular disease risk factors, and metabolic syndrome and its components in patients with psoriasis aged 30 to 49 years. Postepy Dermatologii I Alergologii. 2015; 32:290-5. https://doi.org/10.5114/pdia.2014.40966.

46. Ribeiro Baeta IG, Bittencourt FV, Gontijo B, Andrade Goulart EM. Comorbidities and cardiovascular risk factors in patients with psoriasis. Anais Brasileiros De Dermatologia. 2014; 89:735-44. https://doi.org/10.1590/ abd1806-4841.20142874.

47. Thaci D, Philipp S, Girolomoni G, You R, Fox T. Secukinumab treatment demonstrates a neutral effect on the lipid profile of subjects with moderate-to-severe plaque psoriasis in the 52-week randomized, double-blind, placebo-controlled, phase III FIXTURE study. British Journal of Dermatology. 2014; 171:E157-E8.

48. Topychkanova EP, Filimonkova NN, Kokhan MM, Levchik NK, Kiseleva NV, Sheffer AI. Cytokine profile and cholesterol levels in psoriasis patients with different body mass indices. Klinicheskaya dermatologiya i venerologiya. 2014; 12:11-7.

49. Borodzicz S, Rudnicka L, Mirowska-Guzel D, CudnochJedrzejewska A. The role of epidermal sphingolipids in dermatologic diseases. Lipids in Health and Disease. 2016; 15. https://doi.org/10.1186/s12944-016-0178-7.

50. Grimminger F, Mayser P. Lipid Mediators, Free FattyAcids And Psoriasis. Prostaglandins Leukotrienes and Essential Fatty Acids. 1995; 52:1-15. https://doi. org/10.1016/0952-3278(95)90090-x.

51. Kutlubay Z, Tuzun Y, Wolf R, Engin B. Inflammatory Lipid Mediators in Common Skin Diseases. Skinmed. 2016; 14:23-7.

52. Honda T, Kabashima K. Lipid Mediators and Skin Diseases. In: Yokomizo T and Murakami M, eds. Bioactive Lipid
Mediators: Current Reviews and Protocols. (Tokyo: Springer Japan), 2015. 303-14.

53. St-Pierre MV, Kullak-Ublick GA, Hagenbuch B, Meier PJ. Transport of bile acids in hepatic and non-hepatic tissues. Journal of Experimental Biology. 2001; 204:1673-86.

54. Claudel T, Staels B, Kuipers F. The Farnesoid X receptor - A molecular link between bile acid and lipid and glucose metabolism. Arteriosclerosis Thrombosis and Vascular Biology. 2005; 25:2020-31. https://doi.org/10.1161/01. ATV.0000178994.21828.a7.

55. Chiang JYL. Bile acid regulation of hepatic physiology III. Bile acids and nuclear receptors. American Journal of Physiology-Gastrointestinal and Liver Physiology. 2003; 284:G349-G56. https://doi.org/10.1152/ajpgi.00417.2002.

56. Davis RA, Miyake JH, Hui TY, Spann NJ. Regulation of cholesterol-7 alpha-hydroxylase: BAREly missing a SHP. Journal of Lipid Research. 2002; 43:533-43.

57. Gyurcsovics K, Bertok L. Role of bile acids and endotoxins in the pathogenesis and therapy of psoriasis. Orvosi hetilap. 2000; 141:915-7.

58. Bylesjo M. Extracting meaningful information from metabonomic data using multivariate statistics. Methods in molecular biology (Clifton, NJ). 2015; 1277:137-46. https:// doi.org/10.1007/978-1-4939-2377-9_11.

59. Wishart DS, Tzur D, Knox C, Eisner R, Guo AC, Young N, Cheng D, Jewell K, Arndt D, Sawhney S, Fung C, Nikolai L, Lewis M, et al. HMDB: the human metabolome database. Nucleic Acids Research. 2007; 35:D521-D6. https://doi. org/10.1093/nar/gk1923.

60. Wishart DS, Knox C, Guo AC, Eisner R, Young N, Gautam B, Hau DD, Psychogios N, Dong E, Bouatra S, Mandal R, Sinelnikov I, Xia J, et al. HMDB: a knowledgebase for the human metabolome. Nucleic Acids Research. 2009; 37:D603-D10. https://doi.org/10.1093/nar/gkn810.

61. Wishart DS, Jewison T, Guo AC, Wilson M, Knox C, Liu Y, Djoumbou Y, Mandal R, Aziat F, Dong E, Bouatra S, Sinelnikov I, Arndt D, et al. HMDB 3.0-The Human Metabolome Database in 2013. Nucleic Acids Research. 2013; 41:D801-D7. https://doi.org/10.1093/nar/gks1065.

62. Gyurcsovics K, Bertok L. Pathophysiology of psoriasis: coping endotoxins with bile acid therapy. Pathophysiology: the official journal of the International Society for Pathophysiology. 2003; 10:57-61. https://doi.org/10.1016/j. pathophys.2003.07.001. 